\title{
Homogenization of random porous materials with low order Virtual Elements
}

\author{
Marco Pingaro* \\ Department of Structural and Geotechical Engineering \\ Sapienza University of Rome \\ Roma, Italy \\ Email: marco.pingaro@uniroma1.it \\ Emanuele Reccia \\ Department of Civil, Environmental and Architectural Engineering \\ University of Cagliari \\ Cagliari, Italy \\ Email: emanuele.reccia@unica.it \\ Patrizia Trovalusci \\ Department of Structural and Geotechical Engineering \\ Sapienza University of Rome \\ Roma, Italy \\ Email: patrizia.trovalusci@uniroma1.it
}

\begin{abstract}
A Fast Statistical Homogenization Procedure (FSHP) based on Virtual Element Method (VEM) - previously developed by the authors [1] - has been successfully adopted for the homogenization of particulate random composites, via the definition of the Representative Volume Element (RVE), and of the related equivalent elastic moduli. In particular, the adoption of virtual elements of degree one for modelling the inclusions provided reliable results for materials with low contrast, defined as the ratio between mechanical properties of inclusions and matrix. Porous media are then here described as bi-material systems in which soft circular inclusions, with a very low value of material contrast, are randomly distributed in a continuous stiffer matrix. Several simulations have been performed by varying the level of porosity, highlighting the effectiveness of FSHP in conjunction with virtual elements of degree one.
\end{abstract}

\section{Introduction}

In recent times, the scientific community paid great attention to the influence of inherent uncertainties on behaviour of systems and recognized the importance of stochastic approaches to engineering problems [2]. Engineering experience has shown that uncertainties are involved not only in the assessment of loading but also in the material and geometric properties of engineering systems $[3,4]$. An example may be the mechanical characterization of complex realistic materials, such as heterogeneous media exhibiting non-periodic internal micro-structure. In this framework, statistical computational methods may be useful to evaluate their overall mechanical properties [5-9].

To this material typology belong porous solids: natural or artificial materials optimally designed for the load they carry and the environment in which they exist, such as porous rocks, cork, bones, sponges, aerogel, porous silicon and many others, that have great diffusion in engineering applications. They are, by their very nature, heterogeneous materials characterized

*Address all correspondence to this author. 
by a random distribution of pores in a dense solid. However, it is possible to treat them as continuous materials taking into account the effective properties depending on the dense solid and the porosity [10]. Their micro-structure may be idealized as two phases: the matrix, that is the solid phase, and soft inclusions (pores), randomly distributed, representing the second phase [11]. The elastic properties of two-phase (solid - pores) porous materials depend on the geometrical nature of the pores - shape and size - as well as on the value of porosity $[12,13]$. The determination of the deformation under stress of porous materials is a long-standing problem of great interest, involving multifield [14-18] and/or multiscale descriptions [19-21].

In this paper, we use a statistical homogenization approach $[22,23]$ to derive mechanical properties - here the bulk modulus and Poisson ratio - of porous materials and to evaluate how they changes in relation to porosity. In the case of materials with random microstructure, the lack of periodicity in the inclusions disposition makes it difficult to perform the homogenization process, with particular reference to the possibility of identifying a Representative Volume Element (RVE) and proper boundary conditions to apply at the RVE boundary. Several approaches have been proposed [24-35], also referred to nonclassical continua [23,36,37]. Among these models, we focus our attention on the possibility of approaching the RVE using finitesize scaling of intermediate control volume elements, named Statistical Volume Elements (SVEs), and proceed to homogenization (e.g. [5]).

In this work we refer to [23], where a homogenization procedure consistent with the Hill-Mandel condition [38] has been coupled with a statistical approach, by which scale-dependent bounds on classical moduli are obtained using Dirichlet and Neumann boundary conditions (BCs) for solving boundary value problems (BVPs). This statistically based homogenization procedure has provided significant results $[36,39,40]$, with particular reference to the debated problem of the convergence in the presence of materials with very low (or very high) contrast [37], defined as the ratio between the elastic moduli of inclusions and matrix $[5,29,41]$.

In particular, we use the development of the above mentioned procedure, the Fast Statistical Homogenization Procedure (FSHP) based on Virtual Element Method (VEM) provided in [1]. In FSHP the statistical procedure is automatized and integrated in a completely in house specifically developed code, that makes it possible to quickly and efficiently perform a high number of parametric analyses. VEM $[42,43]$ is an innovative computational method that find many applications in problems of structural mechanics: from linear [44] to non-linear elasticity [45] and damage [46], as well as in homogenization of heterogeneous materials [47]. Here, the adoption of VEM allows improving the computational efficiency: virtual elements are polygons of any shape with n-nodes, so it is possible to use a single element for each inclusion, providing a significant reduction of the computational burden. In this work we fix the minimum length of the inclusion's edges as a percentage of the inclusions' radius. The minimum length is chosen for generate a sufficiently fine mesh in the matrix part. The mesh plays a relevant role in materials with very high or very low contrast, such as porous materials, for which large windows are needed to determine the RVE size. Other advantages are: capabilities of using hanging nodes, that permit local refinements and coupling different degree elements; robustness to distortion of the elements; perfectly coupling with FEM elements; accuracy because the stiffness matrix is computed in precision machine; easy to implement.

In this paper, low order virtual elements are used: the adoption of virtual elements of order one is particularly suitable for low contrast materials, as porous materials, as shown in [1], because the stresses are concentrated in the stiffer matrix and constant stress/strain over the elements do not affect homogenization results.

Thanks to the opportunity provided by FSHP with VEM, several parametric analyses have be performed to characterize overall mechanical parameters of porous materials and to investigate their sensitivity to porosity [48-50]. Attention is paid both at the identification of the RVE size and at the evaluation of the overall mechanical properties, that vary in relation with porosity. For several values of porosity RVE size is then identified and bulk modulus and Poisson ratio are estimated.

The outline of the paper is as follows. In Section 2 the FSHP is detailed and the basic assumption of VEM are reported, in particular for the case of virtual elements of order 1. Section 3 provides the results of parametric analyses on random porous materials, modelled as two-phase material with soft inclusions. Attention is paid to the definition of the RVE size and to the sensitivity to porosity: how RVE size and mechanical properties change at varying of porosity. In Section 4 some final remarks are presented.

\section{Fast Statistical Homogenization Procedure with VEM}

In this Section, the steps of the automated so-called Fast Statistical Homogenization Procedure (FSHP) [1], based on the statistical homogenization procedure previously developed in [23] and briefly described in Section 1, are detailed. FSHP allows to compute overall homogenized mechanical parameters, in particular the anisotropic elasticity tensor. Boundary Values Problems (BVPs) are solved at microscopic level, where each material phase is characterized by linear elastic isotropic behaviour, applying both Dirichlet and Neumann boundary conditions. At the macroscopic level, a general anisotropic 2D stress-strain relation is derived applying the Hills macrohomogeneity condition. Afterwards, the virtual element of degree one adopted in this work is described. 


\subsection{Constitutive model}

We consider a two-dimensional framework and describe, at the microscopic scale, the heterogeneous porous material as a bi-phase system. In two-phase materials it is useful to define the material contrast as the ratio between the elastic moduli of inclusions, $E_{i}$, and matrix, $E_{m}, c=E_{i} / E_{m}$. When $0<c<1(c=1$ being the case of a homogeneous material) inclusions are softer than the matrix and we refer to low contrast materials, that are suitable to properly represent porous media [11]. The pores are modelled as circular inclusions of diameter $d$ dispersed into continuous matrix (Fig. 1(a)). Furthermore a scale parameter $\delta=L / d$ is introduced, that is the ratio between the side of a square test window $L$, and the diameter $d$ of the inclusions. The analysis are developed within a kinematic linearised framework.

In order to perform homogenization, we consider two levels of the description, the microscopic and the macroscopic level, with governing equations that are formally the same except than for the constitutive law that is not 'a priori' defined at the macroscopic level, but directly descends from the lower level as result of the homogenization procedure. In the following, lower case letters always refer to the microscale, while upper case letters to the macroscale. We assume that at the lower level each material phase is characterized by linear elastic isotropic behaviour with the stress-strain relations:

$$
\sigma_{i j}=\lambda \varepsilon_{k k} \delta_{i j}+2 \mu \varepsilon_{i j},
$$

where $\varepsilon_{i j}$ and $\sigma_{i j}(i, j, k=1,2)$ are the components of the microstrain and microstress tensors, $\lambda$ and $\mu$ are the Lamé constants, and $\delta_{i j}(i, j=1,2)$ the Kronecker symbol. At the macroscopic level, the general anisotropic 2D stress-strain relations, read:

$$
\Sigma_{i j}=C_{i j h k} E_{h k},
$$

where $E_{i j}, \Sigma_{i j}(i, j=1,2)$ are the components of the macrostrain and macrostress tensors and $C_{i j h k}(i, j, h, k=1,2)$ the components of the macroscopic elastic tensor, corresponding to the equivalent moduli obtained via a homogenization procedure based on the Hill's macrohomogeneity condition [38]:

$$
\Sigma_{i j} E_{i j}=\frac{1}{A_{\delta}} \int_{\mathcal{B}_{\delta}} \sigma_{i j} \varepsilon_{i j} d A
$$

which states the equivalence between the stress density power in the macroscopic model and the mean power evaluated at the microscopic level within a domain (test window), $\mathcal{B}_{\delta}$, occupying a region of area $A_{\delta}$.

The set of Dirichlet and Neumann boundary conditions (BCs) to apply, at the microscale, on the boundary $\partial \mathcal{B}_{\delta}$, for the solution of the BVPs, directly derive from the fulfilment of the macrohomogeneity condition (3). The Dirichlet BCs are:

$$
u_{i}=E_{i j} x_{j}, \text { on } \partial \mathcal{B}_{\delta},
$$

$u_{i}$ being the components of the displacement vector and $x_{j}(i, j=1,2)$ the $j$-th coordinate of the generic point on the boundary, $\partial \mathcal{B}_{\delta}$, with respect to a reference system with origin in the geometric center of the test window $\mathcal{B}_{\delta}$; while the Neumann $\mathrm{BCs}$ are:

$$
t_{i}=\Sigma_{i j} n_{j}, \text { on } \partial \mathcal{B}_{\delta},
$$

where $t_{i}$ are the components of the traction vector and $n_{j}$ the component of the outward normal to $\partial \mathcal{B}_{\delta}(i, j=1,2)$.

\subsection{Statistical Homogenization}

The homogenization procedure for defining the constitutive response of random heterogeneous material requires the definition of the size of a Representative Volume Element (RVE) larger than the microscale characteristic length, corresponding to the diameter of inclusions, $d$.

According to the approach presented in [23], based on the approach proposed in [22, 25], the presented procedure requires the statistical definition of a number of realizations, called Statistical Volume Elements (SVEs), of the possible microstructure, sampled in a Monte Carlo sense, which allows determining series of scale-dependent upper and lower bounds for the overall elastic moduli and to approach the RVE size, $\delta_{R V E}$, using a statistical criterion to stop the procedure when the results in terms of average elastic moduli do not change within the selected tolerance interval.

All the steps of the homogenization procedure are completely integrated in the FSHP. The entire computational stochastic homogenization procedure is structured as follows and resumed in the Flow chart in Fig. 2. 


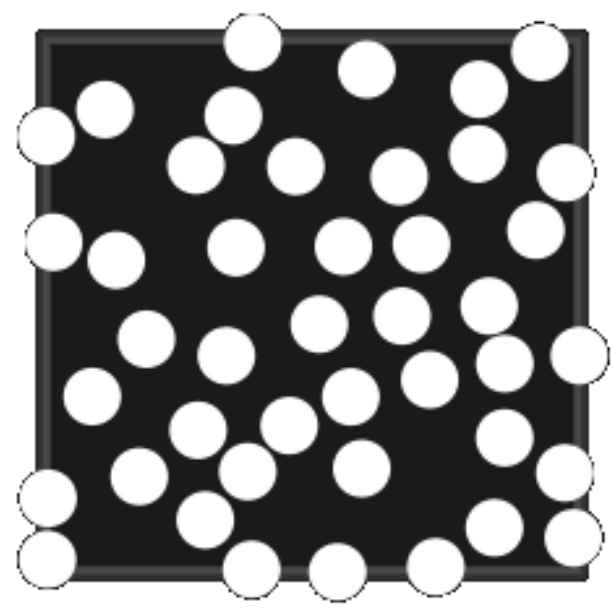

(a)

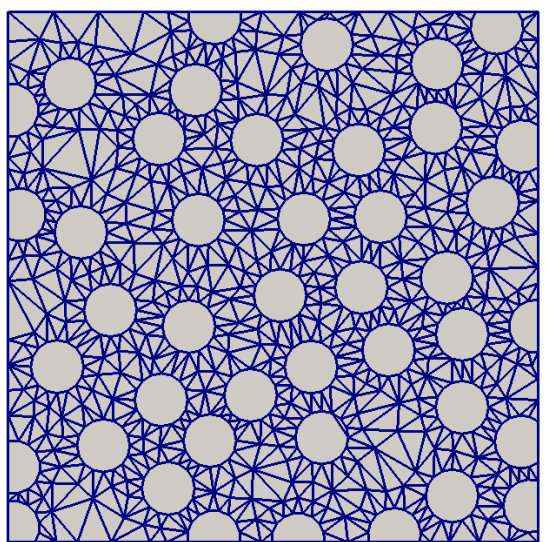

(b)

Fig. 1. Random realization of the microstructure (a) and related mesh (b)

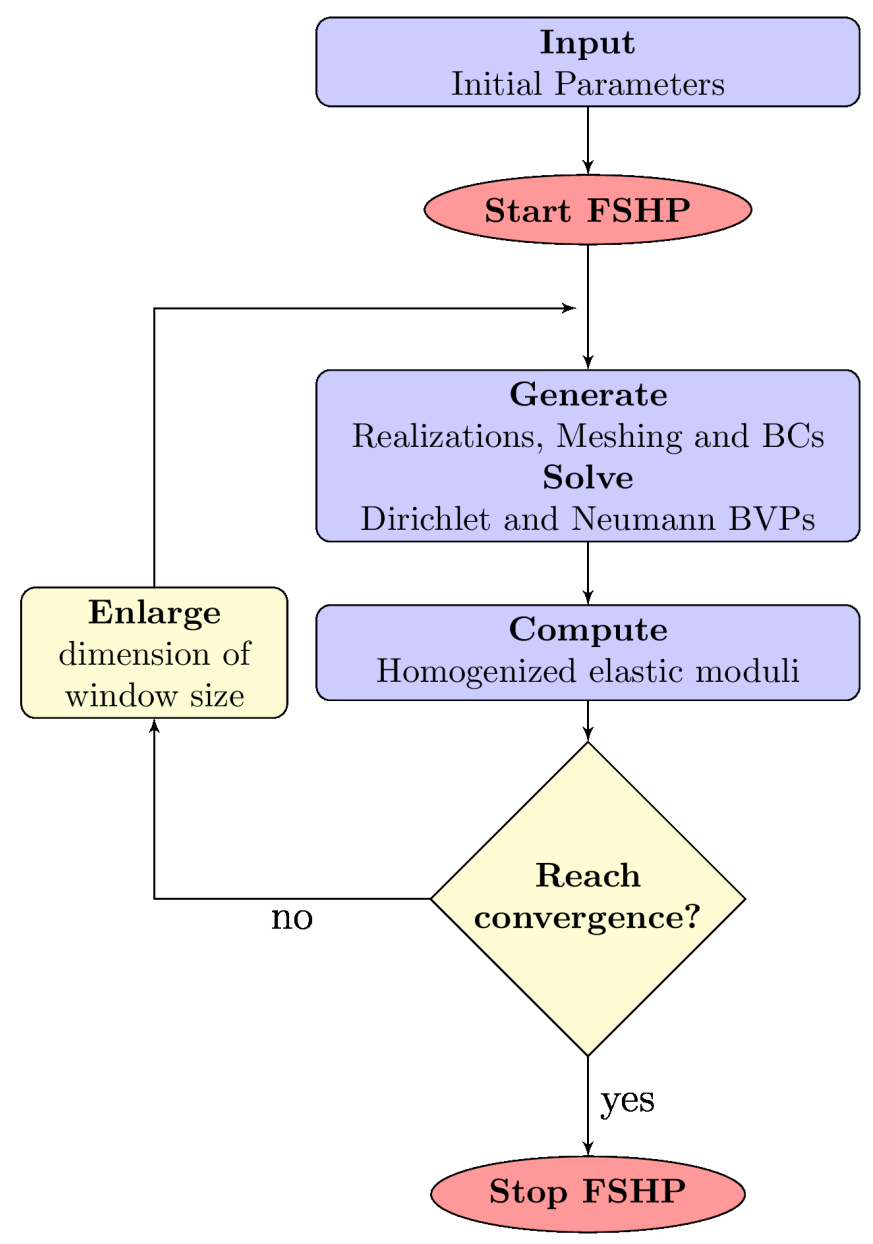

Fig. 2. Flow chart of Fast Statistical Homogenization Procedure (FSHP).

Step 1 Input: set the nominal volume fraction of the medium $(\rho \leq 40 \%)$ as the ratio between the total area of the inclusions and the area of a test window. The particles are disk-shaped inclusions, with diameter $d$, randomly distributed on the base of a Poisson point field process with 'hard-core' distance, $s$, between the particles, fixed in order to avoid very narrow necks between inclusions. Define the dimensionless scale factor $\delta=L / d$. Fix the mechanical parameters of 
each phase: Young modulus of inclusions and matrix, $E_{i}$ and $E_{m}$; Poisson coefficient, $v_{i}$ and $v_{m}$. Set the minimum number of simulations for convergence, $N^{l i m}$, and a tolerance parameter, Tol, based on data dispersion, as defined in above.

Step 2 Input: initialize the window size, $L=L_{0}$, and number of simulations, $N=N_{0}$.

Step 3 Realizations: for each window size determine the number of inclusions (Poisson random variable) via simulations by means of Knuth's algorithm, where the Poisson's distribution is characterized by the parameter $p_{\delta}=\left(\rho L^{2}\right) /[\pi(d / 2+$ $\left.s / 2)^{2}\right]$. Then simulate random dispositions of disks' centres, according to the uniform distribution, that are the realizations (SVEs) of portions of the random medium. Each realization is supposed to be independent from any previous one. An example of realization of the miscrostructure and the related generated mesh is shown in Fig. 1(a).

Step 4 Generate/Solve: for each SVE, generate the relative mesh (Fig. 1(b)) and solve both the Dirichlet (Equation 4) and Neumann (Equations 5) BVP, and compute the homogenized constitutive parameters.

Step 5 Compute: compute the average bulk modulus, $\overline{\mathbb{K}}_{\delta}$, the relative standard deviation $\sigma\left(\overline{\mathbb{K}}_{\delta}\right)$ and variation coefficient $C V\left(\overline{\mathbb{K}}_{\delta}\right)$. Then compute $N_{i}=\left(1.96 \sigma\left(\overline{\mathbb{K}}_{\delta}\right) /\left(\overline{\mathbb{K}}_{\delta} T o l\right)^{2}\right.$, which ensures that the confidence interval of the average homogenized constitutive parameter set at $95 \%$, evaluated over the normal standard distribution, is within the tolerance allowed, Tol. Repeat Steps 3-4 until $N_{i}<N^{\text {lim }}$.

Step 6 Checking: if the number of realizations necessary for ensuring the requirement at Step 5 is small enough, stop the procedure. We choose as the number of realizations necessary the most unfavourable number between those obtained by solving BVPs of Neumann or Dirichlet. Otherwise choose an increased value of $\delta$ and go to Step 3 .

The fulfilment of the requirement at Step 6 means that the values of the homogenized constitutive coefficients are distributed around their averages with a vanishing variation coefficient, and that the RVE size is achieved. The effective constitutive moduli are finally estimated as the mean values between the Dirichlet (upper) and Neumann (lower) bounds at the convergence window (RVE).

The statistical convergence criterion adopted is based on the $95 \%$ over the Normal Standard distribution, which provides the number $\mathrm{N}$ at which is possible to stop the simulations for a given window size $\delta$. When this number is small enough, the average values of the effective moduli converge and the RVE size is achieved. This circumstance also corresponds to reaching the minimum window size $\delta_{R V E}$ for which the estimated homogenized moduli remain constant, within a tolerance interval less than $0.5 \%$ for both the Dirichlet and Neumann solutions. The minimum number of simulations, $N^{l i m}$, and the tolerance parameter, $\mathrm{Tol}$, are chosen in order to define a narrow confidence interval for the average and to obtain a reliable convergence criterion. The choice is discretionary, values are assumed depending on the data dispersion. All these details are specified in [23].

The FSHP permits the automatic cutting of the inclusions over the limit of the windows' edges, accounting for the presence of inclusions that randomly cross the windows' edges as required by the randomness of the medium. It is worth noting that to neglect the presence of inclusions that intersect the windows' edges, a less realistic hypothesis widely used in literature, provides results significantly different from the results obtained taking into account cut inclusions at the windows' edges [36]. Furhermore, the mesh corresponding to each realization of the microstructure has been optimized using VEM methodology, that permits to adopt single virtual element for the inclusions (reduction of degrees of freedom) and triangular virtual elements for the matrix (Fig. 1(b)). In order to take into account the stress gradients in the so-called 'hard core regions', the mesh is fine near the inclusions and coarse away from the inclusions. The FSHP allow us to very efficiently solve the series (hundred) of BVPs and to rapidly converge to the RVE solution.

\subsection{Virtual Element Method}

In this section we recall the weak formulation of the classical linear elastic problem and describe the related virtual element space of degree $1[42,43]$ as well as the construction of the bilinear form resulting from the weak form.

In the following we use the Voigt notation, suited to the proposed formulation.

\subsubsection{The linear elastic problem}

Let $\Omega$ be a two-dimensional domain, and let $\Gamma$ be its boundary. We consider the deformation problem of a linearly elastic body subjected to the volume force, represented by the vector $\boldsymbol{f} \in\left(L^{2}(\Omega)\right)^{2}, \boldsymbol{f}=\left\{f_{1}, f_{2}\right\}^{T}$ (within the standard Lebesgue space), and given boundary conditions, under the hypothesis of small deformations. We use homogeneous Dirichlet boundary conditions and consider the Sobolev space, $\boldsymbol{V}:=\left(H_{0}^{1}(\Omega)\right)^{2}$, of the admissible displacement fields, represented by the vector $\boldsymbol{v}$. Furthermore we represent the strain as the vector $\boldsymbol{\varepsilon}=\left\{\varepsilon_{11}, \varepsilon_{22}, \varepsilon_{12}\right\}^{T}$ associated to the displacement field vector $\boldsymbol{u}=\left\{u_{1}, u_{2}\right\}^{T}:$ 


$$
\boldsymbol{\varepsilon}(\boldsymbol{u})=\boldsymbol{S} \boldsymbol{u} \quad \text { with } \boldsymbol{S}=\left[\begin{array}{cc}
\partial_{x} & 0 \\
0 & \partial_{y} \\
\partial_{y} & \partial_{x}
\end{array}\right]
$$

where $\partial_{(\cdot)}$ denotes the partial derivative with respect to the $(\cdot)$-coordinate.

The weak form of the linear elastostatic problem, provided by the virtual work principle, reads:

$$
\left\{\begin{array}{l}
\text { Find } \boldsymbol{u} \in \boldsymbol{V} \text { such that : } \\
a(\boldsymbol{u}, \boldsymbol{v})=<\boldsymbol{f}, \boldsymbol{v}>\boldsymbol{v} \in \boldsymbol{V}
\end{array}\right.
$$

where:

$$
\begin{aligned}
& a(\boldsymbol{u}, \boldsymbol{v})=\int_{\Omega} \boldsymbol{\varepsilon}(\boldsymbol{v})^{T} \boldsymbol{C} \boldsymbol{\varepsilon}(\boldsymbol{u}) d \Omega=\int_{\Omega}(\boldsymbol{S} \boldsymbol{v})^{T} \boldsymbol{C S} \boldsymbol{u} d \Omega \\
& <\boldsymbol{f}, \boldsymbol{v}>=\int_{\Omega} \boldsymbol{f}^{T} \boldsymbol{v} d \Omega
\end{aligned}
$$

where $\boldsymbol{C}$ is the $3 \times 3$ elastic tensor.

\subsubsection{Virtual element formulation}

In this subsection we introduce the virtual element discretization used in the following simulations. In order to approximate the solution of the problem (7) we consider a decomposition $\mathcal{T}_{h}$ of the domain $\Omega$ into non overlapping polygonal elements $E$. In the following, we denote by $e$ the straight edges of the mesh $\mathcal{T}_{h}$ and, for all $e \in \partial E, \boldsymbol{n}_{i}$ denotes the outward unit normal vector to $e_{i}$ (Fig. 3(a)). The symbol $n_{e}$ represents the number of the edges of the polygon $E$.

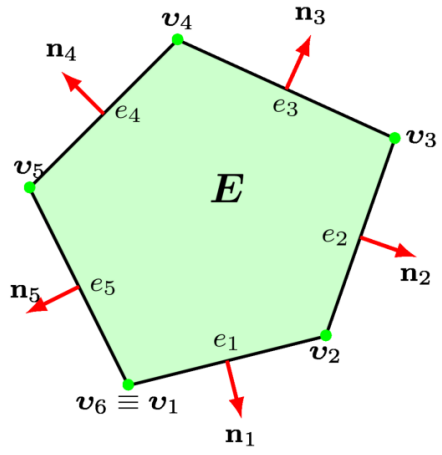

(a)

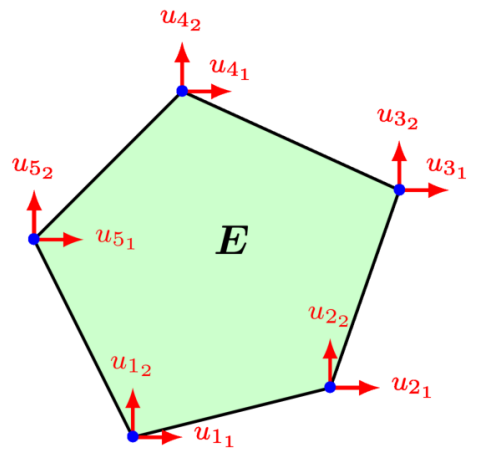

(b)

Fig. 3. Example of virtual element, $E$, with the relative nodes and edges numeration (a) and degrees of freedom of the virtual element of degree $1(b)$.

Let $k$ be an integer $\geq 1$. Let us denote by $P_{k}(\Omega)$ the space of polynomials, living on the set $\Omega \subseteq \mathbb{R}^{2}$, of degree less than or equal to $k$.

By the discretization introduced, it is possible to write the bilinear form (7), as in the finite element methodology, in the following way:

$$
a(\boldsymbol{u}, \boldsymbol{v})=\sum_{E \in \mathcal{T}_{h}} a^{E}(\boldsymbol{u}, \boldsymbol{v}) \quad \forall \boldsymbol{v} \in \boldsymbol{V}
$$

The discrete virtual element space, $\boldsymbol{V}_{h}$, is:

$$
\boldsymbol{V}_{h}:=\left\{\boldsymbol{v} \in \boldsymbol{V}:\left.\boldsymbol{v}\right|_{E} \in \boldsymbol{V}_{h \mid E} \forall E \in \mathcal{T}_{h}\right\}
$$


where the local space, $\boldsymbol{V}_{h \mid E}:=\left[V_{h \mid E}\right]^{2}$. For the virtual element of degree $k=1 V_{h \mid E}$ is defined as:

$$
V_{h \mid E}:=\left\{\boldsymbol{v}_{h} \in H^{1}(E) \cap C^{0}(E): \triangle \boldsymbol{v}_{h} \in\{0\},\left.\boldsymbol{v}_{h}\right|_{e} \in P_{1}(e) \forall e \in \partial E\right\}
$$

The dimension of the space $\boldsymbol{V}_{h \mid E}$ then is:

$$
m=\operatorname{dim}\left(\boldsymbol{V}_{h \mid E}\right)=2 n_{e} .
$$

We can observe that, in contrast to the standard finite element approach, the local space $\boldsymbol{V}_{h \mid E}$ is not fully explicit. Moreover $\boldsymbol{v}_{h}$ is a polynomial of degree 1 on each edge $e$ of $E$ and globally continuous on $\partial E$.

The problem (7) restricted to the discrete space $\boldsymbol{V}_{h}$ becomes:

$$
\left\{\begin{array}{l}
\text { Find } \boldsymbol{u}_{h} \in \boldsymbol{V}_{h} \text { such that } \\
a_{h}\left(\boldsymbol{u}_{h}, \boldsymbol{v}_{h}\right)=<\boldsymbol{f}, \boldsymbol{v}_{h}>\quad \forall \boldsymbol{v}_{h} \in \boldsymbol{V}_{h},
\end{array}\right.
$$

where $a_{h}(\cdot, \cdot): \boldsymbol{V}_{h} \times \boldsymbol{V}_{h} \rightarrow \mathbb{R}$ is the discrete bilinear form approximating the continuous form $a(\cdot, \cdot)$ and, $<\boldsymbol{f}, \boldsymbol{v}_{h}>$ is the term approximating the virtual work of external load. In this work the body force is null and then we do not report any detail for the implementation. The discrete bilinear form is constructed element by element as

$$
a_{h}\left(\boldsymbol{u}_{h}, \boldsymbol{v}_{h}\right)=\sum_{E \in \mathcal{T}_{h}} a_{h}^{E}\left(\boldsymbol{u}_{h}, \boldsymbol{v}_{h}\right) \quad \forall \boldsymbol{u}_{h}, \boldsymbol{v}_{h} \in \boldsymbol{V}_{h}
$$

By the above definition of the local space (11) the following important observations can be made:

- the functions $\boldsymbol{v}_{h} \in \boldsymbol{V}_{h \mid E}$ are explicitly known on $\partial E$ (linear functions);

- the functions $\boldsymbol{v}_{h} \in \boldsymbol{V}_{h \mid E}$ are not explicitly known inside the element $E$;

- $\left(P_{1}(E)\right)^{2} \subseteq \boldsymbol{V}_{h \mid E}$.

The related degrees of freedom for the space $\boldsymbol{V}_{h \mid E}$ are $2 n_{e}$ point-wise values $\boldsymbol{v}_{h}\left(v_{i}\right) i=1,2, \ldots, n_{e}$, where $v_{i}$ is the $i$-th corner of $E$.

\subsubsection{Projection operator and construction of the stiffness matrix}

In accordance to [51] we define the projector operator of the strain as:

$$
\begin{aligned}
& \Pi: \boldsymbol{V}_{h \mid E} \rightarrow P_{0}(E)_{s y m}^{2 \times 2} \\
& \boldsymbol{v}_{h} \rightarrow \Pi\left(\boldsymbol{v}_{h}\right)
\end{aligned}
$$

In particular the projector operator respects the following property of orthogonality:

$$
\int_{E} \Pi\left(\boldsymbol{v}_{h}\right)^{T} \hat{\boldsymbol{\varepsilon}} d E=\int_{E} \boldsymbol{\varepsilon}\left(\boldsymbol{v}_{h}\right)^{T} \hat{\boldsymbol{\varepsilon}} d E \quad \forall \hat{\boldsymbol{\varepsilon}} \in P_{0}(E)_{s y m}^{2 \times 2} .
$$

As in the finite element method, we define the vectors $\boldsymbol{v}_{h} \in \boldsymbol{V}_{h \mid E}$ and $\hat{\boldsymbol{\varepsilon}} \in P_{0}(E)_{\text {sym }}^{2 \times 2}$ using the degrees of freedom $\overline{\boldsymbol{v}}_{h} \in \mathbb{R}^{2 n_{e}}$, $\hat{\boldsymbol{\varepsilon}} \in \mathbb{R}^{3}:$

$$
\left\{\begin{array}{l}
\boldsymbol{v}_{h}=\boldsymbol{N}^{V} \overline{\boldsymbol{v}}_{h} \\
\hat{\boldsymbol{\varepsilon}}=\boldsymbol{N}^{u} \overline{\boldsymbol{\varepsilon}}
\end{array}\right.
$$

The matrices $\boldsymbol{N}^{u}$ and $\boldsymbol{N}^{V}$ are

$$
\boldsymbol{N}^{u}=\left[\begin{array}{lll}
1 & 0 & 0 \\
0 & 1 & 0 \\
0 & 0 & 1
\end{array}\right], \quad \boldsymbol{N}^{V}=\left[\begin{array}{ccccc}
\phi_{1} & 0 & \cdots & \phi_{n_{e}} & 0 \\
0 & \phi_{1} & \cdots & 0 & \phi_{n_{e}}
\end{array}\right]
$$


where $\phi_{i}$ is the standard $i-$ th shape function. The projection operator in matrix is:

$$
\Pi\left(\boldsymbol{v}_{h}\right)=\boldsymbol{N}^{u} \bar{\Pi} \overline{\boldsymbol{v}}_{h},
$$

with $\bar{\Pi} \in \mathbb{R}^{3 \times m}$.

By putting Equations (17-19) into the Equation (16) we obtain:

$$
\int_{E}\left(\boldsymbol{N}^{u} \bar{\Pi} \overline{\boldsymbol{v}}_{h}\right)^{T} \boldsymbol{N}^{u} \overline{\boldsymbol{\varepsilon}} d E=\int_{E}\left[\boldsymbol{\varepsilon}\left(\boldsymbol{N}^{V} \overline{\boldsymbol{v}}_{h}\right)\right]^{T} \boldsymbol{N}^{u} \overline{\boldsymbol{\varepsilon}} d E \quad \forall \overline{\boldsymbol{\varepsilon}} \in \mathbb{R}^{3}
$$

Integrating by part the right hand side we obtain:

$$
\int_{E}\left(\boldsymbol{N}^{u} \bar{\Pi} \overline{\boldsymbol{v}}_{h}\right)^{T} \boldsymbol{N}^{u} \overline{\boldsymbol{\varepsilon}} d E=\int_{\partial E}\left(\boldsymbol{N}^{V} \overline{\boldsymbol{v}}_{h}\right)^{T}\left(\boldsymbol{N}_{E} \boldsymbol{N}^{u} \overline{\boldsymbol{\varepsilon}}\right) d \partial E \quad \forall \overline{\boldsymbol{\varepsilon}} \in \mathbb{R}^{3},
$$

where $\boldsymbol{N}_{E}$ contains the components of the outward normal $\boldsymbol{n}=\left\{n_{1}, n_{2}\right\}^{T}$

$$
\boldsymbol{N}_{E}=\left[\begin{array}{ccc}
n_{1} & 0 & n_{2} \\
0 & n_{2} & n_{1}
\end{array}\right]
$$

After some algebra:

$$
\overline{\boldsymbol{\varepsilon}}^{T}\left\{\int_{E}\left(\boldsymbol{N}^{u}\right)^{T} \boldsymbol{N}^{u} d E\right\} \bar{\Pi} \overline{\boldsymbol{v}}_{h}=\overline{\boldsymbol{\varepsilon}}^{T}\left\{\int_{\partial E}\left(\boldsymbol{N}_{E} \boldsymbol{N}^{u}\right)^{T} \boldsymbol{N}^{V} d \partial E\right\} \overline{\boldsymbol{v}}_{h} \quad \forall \overline{\boldsymbol{\varepsilon}} \in \mathbb{R}^{3}
$$

Finally the local projector operator $\bar{\Pi}$ is:

$$
\bar{\Pi}=\boldsymbol{G}^{-1} \boldsymbol{B},
$$

where:

$$
\boldsymbol{G}=\int_{E}\left(\boldsymbol{N}^{u}\right)^{T} \boldsymbol{N}^{u} d E, \quad \boldsymbol{B}=\int_{\partial E}\left(\boldsymbol{N}_{E} \boldsymbol{N}^{u}\right)^{T} \boldsymbol{N}^{V} d \partial E,
$$

We can observe that the matrix $\boldsymbol{G} \in \mathbb{R}^{3 \times 3}$ is computed by knowing the area of the elements $|E|$ :

$$
\boldsymbol{G}=\left[\begin{array}{ccc}
|E| & 0 & 0 \\
0 & |E| & 0 \\
0 & 0 & |E|
\end{array}\right]
$$

and the matrix $\boldsymbol{B} \in \mathbb{R}^{3 \times m}$ using the degrees of freedom of the element:

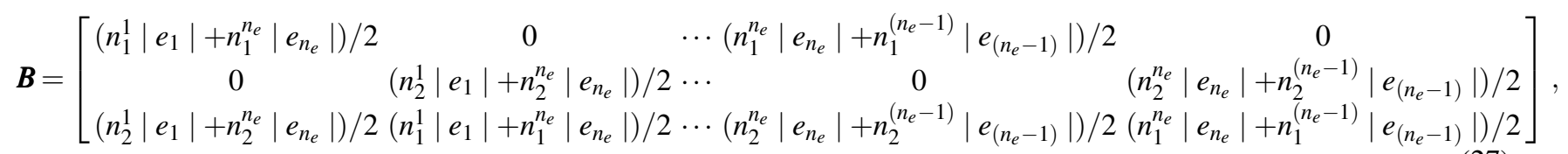

where $\left|e_{i}\right|$ is the length of the $i$-th edge of the element $E$ and $n_{j}^{i}\left(i=1, \cdots, n_{e} ; j=1,2\right)$ is the $i$-th outward normal of components $j$.

In the virtual element strategy we approximate the bilinear form of Equation (14) as follow

$$
a_{h}^{E}\left(\boldsymbol{u}_{h}, \boldsymbol{v}_{h}\right)=\int_{E}\left[\Pi\left(\boldsymbol{v}_{h}\right)\right]^{T} \boldsymbol{C} \Pi\left(\boldsymbol{u}_{h}\right) d E+S^{E}\left(\boldsymbol{u}_{h}, \boldsymbol{v}_{h}\right)=\overline{\boldsymbol{v}}_{h}^{T} \boldsymbol{M} \overline{\boldsymbol{u}}_{h}+\overline{\boldsymbol{v}}_{h}^{T} \boldsymbol{K}_{s} \overline{\boldsymbol{u}}_{h},
$$


where the first term of the right hand side is the consistent term and the second term is the stabilization term.

For computing the consistent term $\boldsymbol{M}$ we use Equation (19) and Equation (8)

$$
\int_{E}\left[\Pi\left(\boldsymbol{v}_{h}\right)\right]^{T} \boldsymbol{C} \Pi\left(\boldsymbol{u}_{h}\right) d E=\overline{\boldsymbol{v}}_{h}^{T} \bar{\Pi}^{T}\left[\int_{E}\left(\boldsymbol{N}^{u}\right)^{T} \boldsymbol{C} \boldsymbol{N}^{u} d E\right] \bar{\Pi} \overline{\boldsymbol{u}}_{h}
$$

where

$$
\boldsymbol{M}=\bar{\Pi}^{T}\left[\int_{E}\left(\boldsymbol{N}^{u}\right)^{T} \boldsymbol{C} \boldsymbol{N}^{u} d E\right] \bar{\Pi}
$$

If the elastic tensor is constant, it is possible to rearrange Equation (30) and obtain:

$$
\boldsymbol{M}=\bar{\Pi}^{T} \boldsymbol{C G} \bar{\Pi} .
$$

Regarding the stabilization term, we adopt the choice introduced in $[42,43]$. First of all, we introduce the scaled coordinates $(\xi, \eta)$ as:

$$
\xi=\frac{x-x_{E}}{h_{E}}, \quad \eta=\frac{y-y_{E}}{h_{E}}
$$

where $\left(x_{E}, y_{E}\right)$ are the coordinates of the centre of $E$ and $h_{E}$ its diameter.

The stabilization term are define as follow

$$
\boldsymbol{K}_{s}=\alpha \operatorname{tr}(\boldsymbol{M})\left[\boldsymbol{I}-\boldsymbol{D}\left(\boldsymbol{D}^{T} \boldsymbol{D}\right)^{-1} \boldsymbol{D}^{T}\right]
$$

where $\alpha \in R$ and in this work is set equal to $1 / 2$. The matrix $\boldsymbol{D}$ contains the scaled coordinates at the nodes of the element:

$$
\boldsymbol{D}=\left[\begin{array}{cccccc}
1 & 0 & \xi_{1} & 0 & \eta_{1} & 0 \\
0 & 1 & 0 & \xi_{1} & 0 & \eta_{1} \\
\vdots & \vdots & \vdots & \vdots & \vdots & \vdots \\
1 & 0 & \xi_{n_{e}} & 0 & \eta_{n_{e}} & 0 \\
0 & 1 & 0 & \xi_{n_{e}} & 0 & \eta_{n_{e}}
\end{array}\right]
$$

\section{Numerical analyses}

In this section, the Fast Statistical Homogenization Procedure (FSHP) based on the low order virtual elements is applied to the analysis of porous materials, modelled as a two-dimensional two-phase material in which circular soft inclusions are randomly embedded in a stiffer continuous matrix.

FSHP with virtual element of $k=1$ is particularly suitable for analysing low contrast materials [1], and in this paper a very low value of contrast $(c \rightarrow 0)$ is adopted, with the purpose of simulating a material with randomly distributed voids. Referring to the properties adopted in [11], an high value of Poisson coefficient has been adopted for the inclusions. All the mechanical properties are reported in Table 1 , where $E_{m}$ and $v_{m}$ are Young and Poisson modulus for the matrix, and $E_{i}$ and $v_{i}$ are Young and Poisson modulus for the inclusions, respectively.

\begin{tabular}{lllll}
\hline$E_{m}$ & $E_{i}$ & $c$ & $v_{m}$ & $v_{i}$ \\
\hline 10000 & 1 & $10^{-4}$ & 0.30 & 0.49 \\
\hline
\end{tabular}

Table 1. Mechanical properties adopted

The procedure is applied to the definition of the RVE size in case of random porous materials and to the evaluation of their mechanical properties. Initially the procedure is used keeping constant the porosity, defined as the volume fraction of 
pores, at a value $\rho=40 \%$, for describing how the RVE size, $\delta_{R V E}$, is identified. Subsequently, exploiting the opportunities provided by FSHP, several parametric analyses are performed by varying the porosity, $\rho$, in the range $0 \% \div 40 \%$. The purpose is twofold: on one hand for identifying $\delta_{R V E}$ and its changes in relation of porosity; on the other hand, to evaluate the sensitivity to porosity of the mechanical properties of homogeneous equivalent continuum materials.

\subsection{RVE definition}

Homogenization of random porous materials, modelled as a very low contrast material, requires an high number of realizations and the adoption of large windows to detect the RVE size, $\delta_{R V E}$, and determine the overall elastic moduli [37]. FSHP consists in solving the Boundary Value Problem (BVP) on a huge number - hundreds of analyses - of test windows of increasing size, $\delta$. BVPs are solved by applying both Dirichlet and Neumann BCs, providing upper and lower bounds for the equivalent elastic moduli respectively. As the windows size increases, the number of simulations needed to obtain the convergence of the results decreases. By referring to Section 2.2 we recall that convergence of the results is reached when the average value of an estimated mechanical parameter does not significantly changes after $N$ simulations. The homogenized elasticity tensor is anisotropic by definition, however, the pores being of circular shape and randomly distributed, after several simulations the homogenized elastic tensor moduli has been found to be substantially isotropic. Then in the following we select the bulk modulus, $\mathbb{K}=2 C_{1122}+C_{1212}$ as representative material parameter to identify.

In Fig. 4, the average value of the bulk modulus obtained after $N$ simulations $\overline{\mathbb{K}}_{n}$ is normalized versus the value obtained after $N-1$ simulations, both for Dirichlet BCs (Fig. 4(a)) and for Neumann BCs (Fig. 4(b)). Two different windows of increasing size $\delta$ are considered as example: $\delta=20$, in blue solid line, and $\delta=30$, in red solid line. It is possible to notice that the value of the ratio $\overline{\mathbb{K}}_{n} / \overline{\mathbb{K}}_{n-1}$ undergoes less variations as the number of simulations $N$ increases, and that, as expected, the number of simulations needed to obtain the convergence decreases as the windows size $\delta$ increases. In the reported example, the convergence is reached for $N \simeq 55$ for $\delta=20$ and $N \simeq 25$ for $\delta=30$ in Dirichlet BVP, while $N \simeq 125$ for $\delta=20$ and $N \simeq 45$ for $\delta=30$ are necessary in the case of Neumann BVP. After reaching the convergence for a given $\delta$, FSHP goes on and larger windows' size, $\delta$, are analysed until the number of simulations needed to obtain convergence, $N$, is small enough (Step 6 in 2.2)

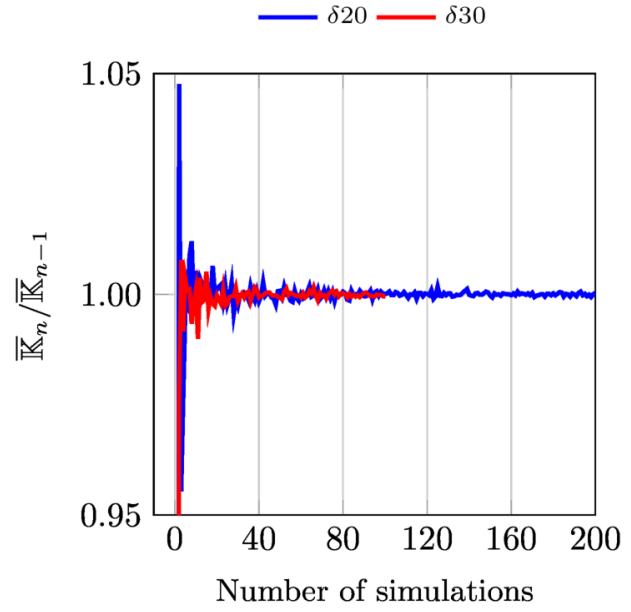

(a) Dirichlet

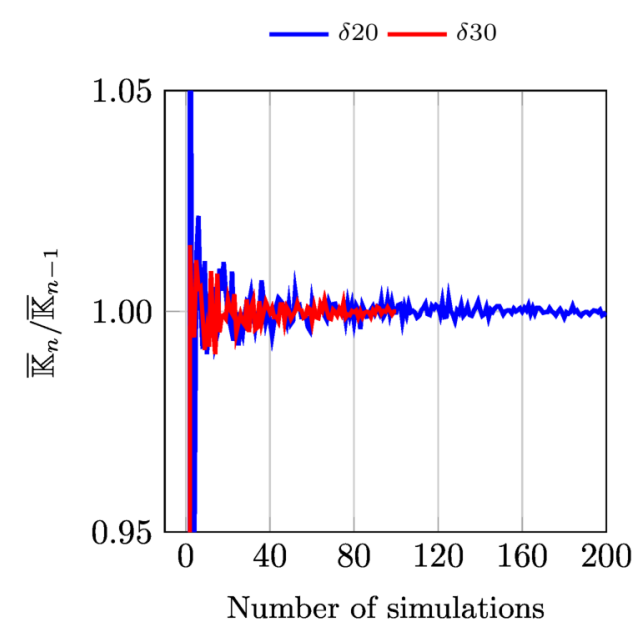

(b) Neumann

Fig. 4. Normalized average bulk modulus $\overline{\mathbb{K}}_{n} / \overline{\mathbb{K}}_{n-1}$ vs the number of simulations

Based on the criterion of the minimum number of simulations needed, the RVE can be reached also for material with very low contrast, such as the porous materials here considered. Note that $\delta_{R V E}$ is then identified as the smallest window size needed to reach the convergence for the BVPs, considering the most unfavourable case between Dirichlet and Neumann BCs. The results obtained by applying Dirichlet and Neumann BCs provide the same trend, however it is possible to notice that the number of simulations needed is smaller in Dirichlet BVP solution. The difference in term of convergence between the two BVPs has been highlighted in [5,22]. In particular, it has been showed that in the case of material with high contrast, where inclusions are stiffer than matrix, Neumann BVP reaches the convergence faster than Dirichlet BVP, while the opposite occurs for low contrast materials $[1,37]$. In the case of porous media, the most unfavourable case is Neumann BVP, meaning that larger windows are needed to reach the convergence value with respect to Dirichlet BVP. In Fig. 5, the number of simulations needed to obtain the convergence for increasing value of windows size $\delta$ is reported. Red solid lines 
are referred to the solution of Dirichlet BVP, while blue solid lines to the solution of Neuman BVP; the same colors are used for Dirichlet and Neumann in the following figures. Dirichlet BCs provides convergence results with a low number of simulations, starting from windows of size $\delta \geq 25$, while larger windows, $\delta>35$, are needed when adopting Neumann BCs. The identification of $\delta_{R V E}$ always requires the solution of the Neumann BVP, resulting, as mentioned above, the most unfavourable case.

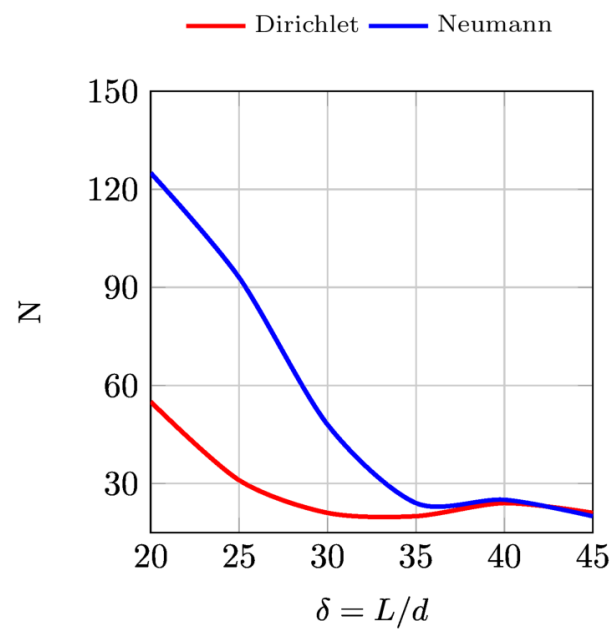

Fig. 5. Number of simulations for reaching the convergence vs window size $\delta$

Fig. 6 reports the average value of the bulk modulus, $\overline{\mathbb{K}}$, normalized with respect to the value estimated at the RVE, $\mathbb{K}_{R V E}$, versus the number of simulations performed for different window sizes, $\delta$, providing a picture of the statistical convergence criterion adopted. $\mathbb{K}_{R V E}$ is evaluated as the mean value between the two values obtained by solving Dirichlet and Neumann BVPs at the convergence window $\delta_{R V E}$. $\delta_{R V E}$ is identified for a window size $\delta \simeq 37$, obtained after about $N \simeq 20$ simulations (Fig. 5). As expected, widening the windows size provides decreasing $\overline{\mathbb{K}}$ in Dirichlet BVP solution (Fig. 6(a)) and raising $\overline{\mathbb{K}}$ in Neumann BVP solution (Fig. 6(b)).

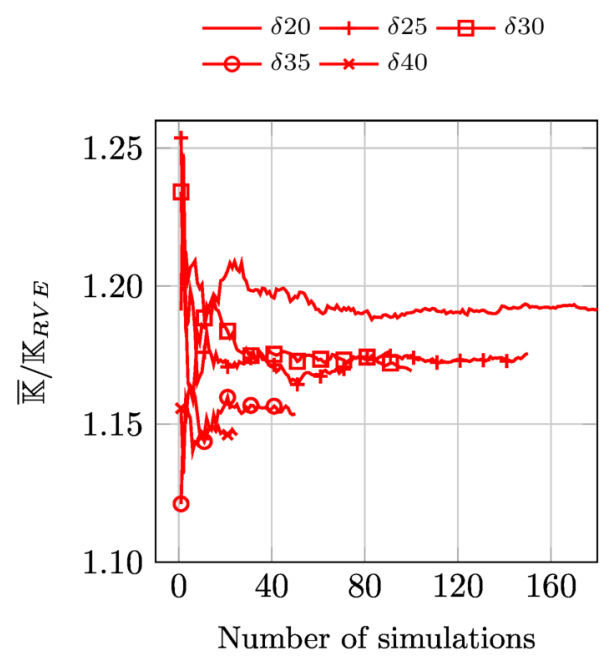

(a) Dirichlet

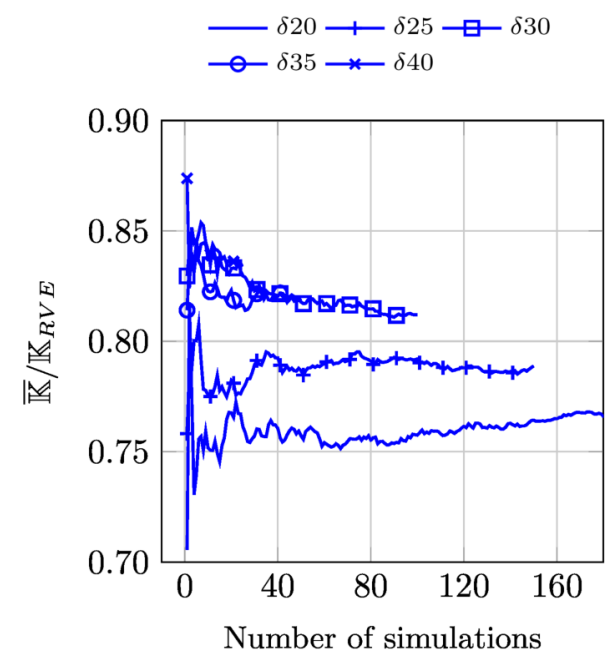

(b) Neumann

Fig. 6. Normalized average bulk modulus $\overline{\mathbb{K}} / \mathbb{K}_{R V E}$ vs window sizes $\delta$

Concerning the convergence trend, as $\delta$ increases the results of Dirichlet and Neuman BVPs tend to get closer to each other, as shown in Fig. 7(a), where the average value of the homogenized bulk modulus normalized with respect to the value 
at $\delta_{R V E}, \overline{\mathbb{K}} / \mathbb{K}_{R V E}$, is plotted versus the scale parameter $\delta$. Even if there is a mismatch between the two solutions, for values of $\delta \geq 37$ they become parallel and $\delta_{R V E}$ is identified.

The differences in terms of convergence trend between Dirichlet and Neumann BVPs solutions also depend on the different dispersion of the results, as shown in Fig. 7(b), where the Coefficient of Variation, $C V$, is plotted versus $\delta$. Note that the convergence is obtained for values of $C V \leq 0.05$ and this occurs for $\delta \geq 25$ in Dirichlet BVP and for $\delta \geq 37$ in Neumann BVP.

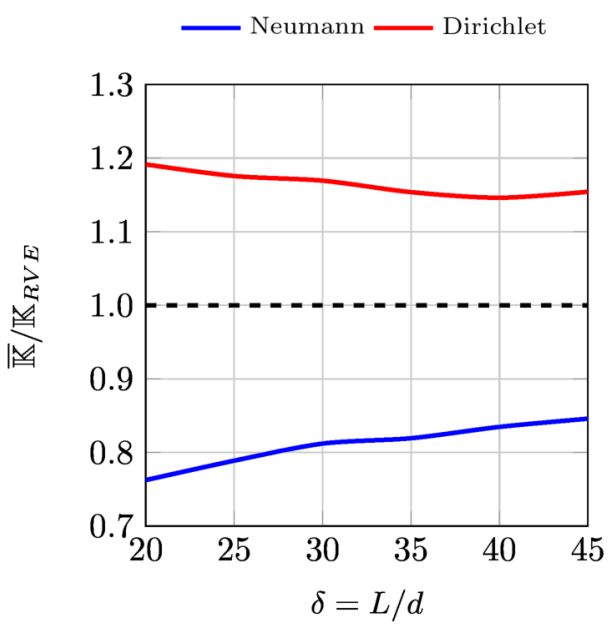

(a) $\overline{\mathbb{K}} / \mathbb{K}_{R V E}$ vs window sizes $\delta$

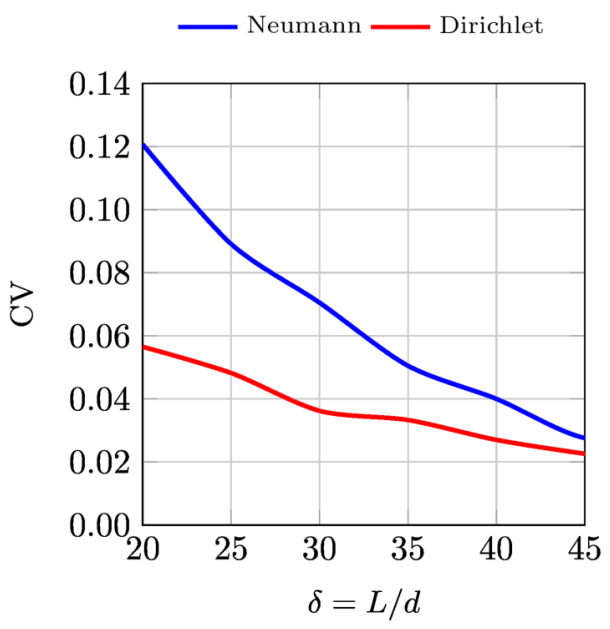

(b) $C V$ vs window sizes $\delta$

Fig. 7. Normalized average bulk modulus $\overline{\mathbb{K}} / \mathbb{K}_{R V E}$ (a) and Coefficient of Variation $C V$ (b) for different window sizes $\delta$

\subsection{Sensitivity to porosity}

Thanks to the opportunities provided by FSHP, several parametric numerical analyses have been performed, with the purpose of evaluating sensitivity to porosity in random porous media. Different values of porosity $0 \div \rho \div 40 \%$ have been considered. In Fig. 8 examples of microstructures for different values of $\rho$ are provided. The performed analyses allowed us to identify RVE size, $\delta_{R V E}$, and the corresponding mechanical properties. Here the attention is focused on the bulk modulus, $\mathbb{K}$, defined in Subsection 3.1, and on Poisson ratio, $v=\left(C_{1122} / C_{1111}\right) /\left(1+\left(C_{1122} / C_{1111}\right)\right)$.

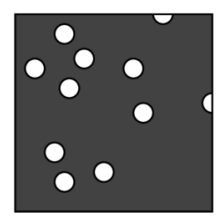

$\rho=5 \%$

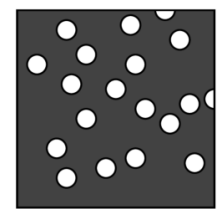

$\rho=20 \%$

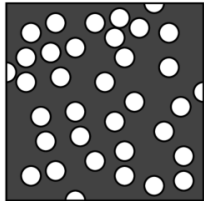

$\rho=40 \%$

Fig. 8. Realizations of micro-structure for different levels of porosity

The results of the parametric analyses performed are reported in the following figures. As above, red color always refers to the solution of Dirichlet BVP, while the blue one to the solution of Neumann BVP. The graphs in Fig. 9 provide a synthetic response of the mechanical parameters variation for different values of porosity.

Fig. 9(a) summarizes the results of the parametric analyses in term of bulk modulus $\mathbb{K}$ versus the porosity $\rho$. As previously obtained, for increasing $\delta$, the two bounds tend to become closer to each other. The value of $\bar{K}$ decreases in Dirichlet solutions while raises in Neumann solutions, as shown in the zoom area of Fig. 9(a) for $\rho=25$, where the values of $\bar{k}$ for increasing $\delta$ are plotted both for Dirichlet (circles) and Neumann (squares) BVPs. As underlined in Section 3.1, Dirichlet BVP reaches convergence for $\delta$ smaller while Neumann BVP requires larger $\delta$ and results are more dispersed. 
For each value of $\rho$, the lowest value of $\bar{K}$ provided by Dirichlet BVPs and the higher value obtained by Neumann BVPs are achieved at $\delta_{R V E}$. The area included between Dirichlet and Neumann BVPs, marked by grey lines pattern, represents the envelope between the two bounds: $\mathbb{K}_{R V E}$ for increasing $\rho$ is obtained as the mean value between the two bounds. As expected, the value of $\mathbb{K}$ decreases as the porosity increases [48]. Apparently the two bounds tend to become closer as $\rho$ increases, however the percentage difference between the bounds and the mean value remains almost constant.

Fig. 9(b) provides the results of the parametric analyses in term of Poisson ratio $\bar{v}$ versus porosity $\rho$. Also in this case, $\bar{v}$ decreases as the porosity increases, as reported in scientific literature [49,50]. It is interesting to point out that in this case, differently from what generally occurs, Neumann BVP solutions provide higher values of $v$ with respect to Dirichlet BVP, becoming the upper bound of the solution. This is probably due to the fact that Neumann BCs are weaker then Dirichlet ones, where transversal contraction is avoided, so that transversal strain is bigger and consequently Poisson's coefficient too.

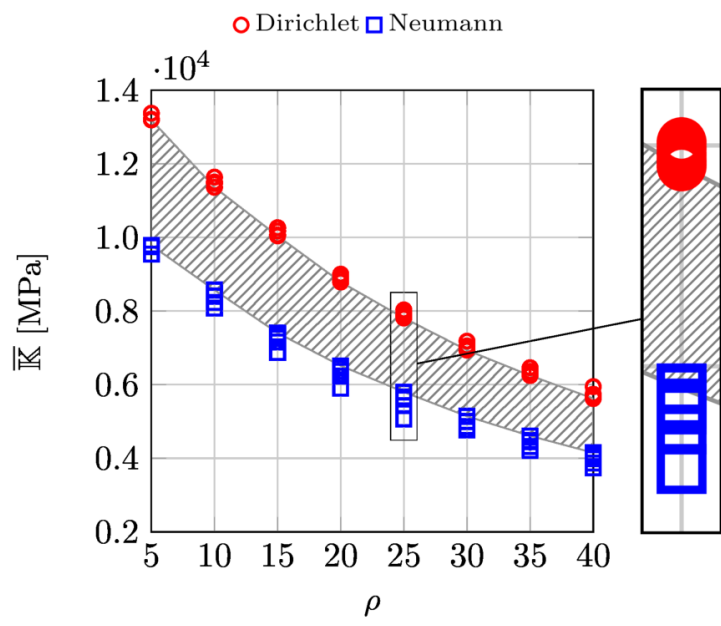

(a) Bulk modulus $\overline{\mathbb{K}}$ for different density $\rho$

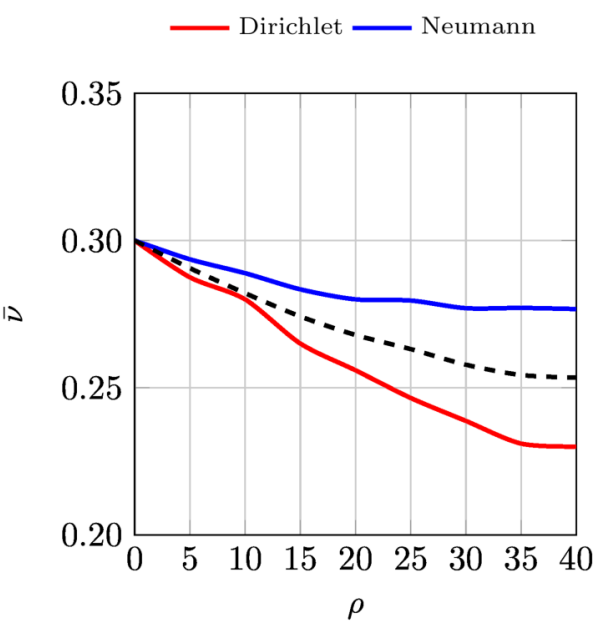

(b) Poisson modulus $\bar{v}$ for different density $\rho$

Fig. 9. Normalized average elastic moduli for different levels of porosity

It is interesting to look out at the RVE size obtained for different values of porosity, (Fig. 10). In the case of $\rho=0$ the material is homogeneous, starting from $\rho \geq 0$ it is necessary to determine a RVE and, as expected, as porosity increases bigger $\delta_{R V E}$ are needed. However, it has been found that the $\delta_{R V E}$ rapidly increases in the range of porosity $1 \div \rho \div 20$, while slowly decreases up to $\rho=25$ and then remain almost constant in the range $25 \div \rho \div 40$. The results in terms of RVE size, $\delta_{R V E}$, are synthesized and reported in Fig. 10, where the values of $\delta=\delta_{R V E}$ are plotted versus the different values of porosity $\rho$ considered. As previously noticed, Dirichlet BVP need smaller windows with respect to Neumann BVP, that has to be considered to define $\delta_{R V E}$ for porous material.

The differences in terms of convergence trend between the two BCs depend on the different dispersion of results, as shown in Fig. 11, where the Coefficient of Variation $C V$ is plotted versus several values of porosity $\rho$ for Dirichlet (Fig. 11(a)) and Neumann BVP (Fig. 11(b)).

\section{Conclusions}

In this work, a fast statistical homogenization procedure (FSHP) has been adopted for the homogenization of random porous materials. Porous material has been modelled as bi-phase material with very low contrasts: disk shaped soft inclusions randomly distributed within a base stiffer matrix. FSHP based on virtual elements of degree 1 allow us to perfectly model porous material [1] and permits to solve high number of simulations as required in homogenization techniques applied to random materials $[23,36,37,40]$. Furthermore, FSHP permits to analyse a series of materials with different porosity and to rapidly identify the relative RVE size and the overall effective moduli of the equivalent homogeneous material.

Some final remarks may be drawn:

- FSHP provides reliable results for the homogenization of porous materials.

- VEM of degree 1 perfectly fits the behaviour of porous materials: stress and strain are constant in low order virtual elements, however this does not have influence on homogenization results in case low contrast materials, as shown in [1].

- The Representative Volume Element (RVE) as a function of the porosity has strongly non-linear behaviour. 


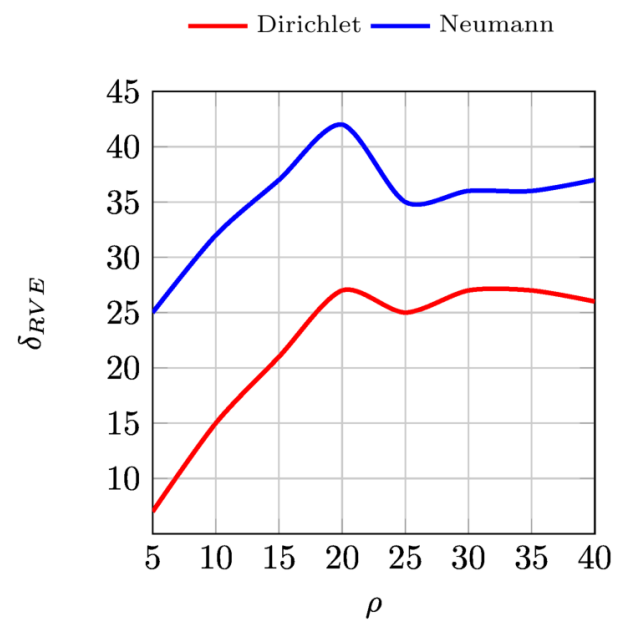

Fig. 10. $\delta_{R V E}$ for different levels of porosity $\rho$

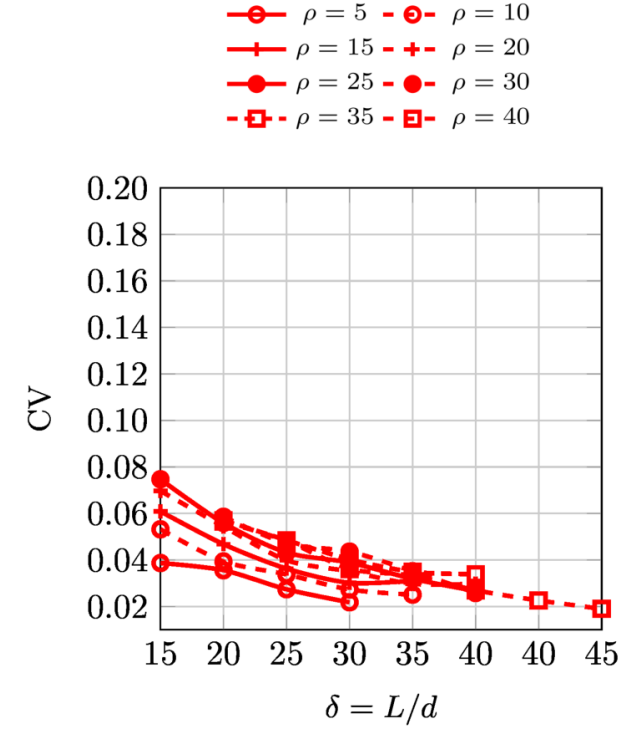

(a) Dirichlet

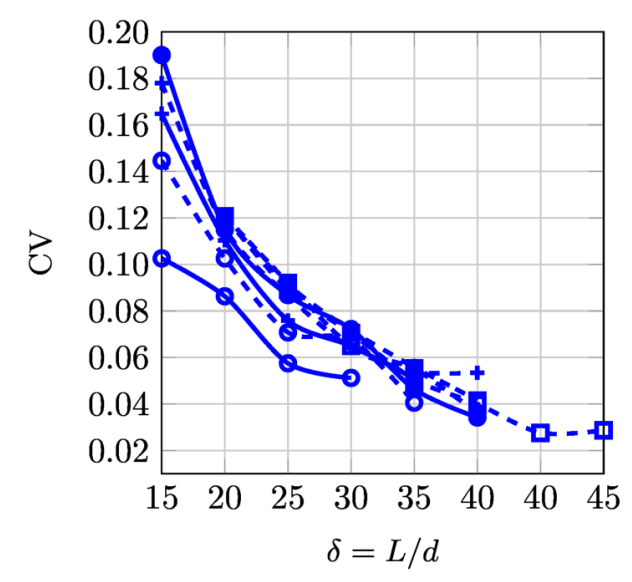

(b) Neumann

Fig. 11. Coefficient of Variation $C V$ for differet levels of porosity $\rho$

- The largest RVE size corresponds to a value of porosity $\rho \simeq 20 \%: \delta_{R V E}$ increases as porosity increases up this value. For higher level of porosity $\delta_{R V E}$ slightly decreases and then remains constant up to the value $\rho=40 \%$, which is the maximum value of porosity.

- Both the homogenized values of the elastic moduli (bulk modulus and Poisson coefficient) decrease as the porosity increases.

- We obtain an inversion between upper and lower bounds for the Poisson coefficient with respect to the material porosity.

Next step of this research is to improve the accuracy of the results by adopting virtual elements higher degree for studying material with hard inclusions dispersed in softer matrix. Moreover, the proposed method will be implemented in order to better describe the behaviour of micro-structured material made of particles of different shape and orientation by adopting enriched non-classical continua descriptions, such as Couple-Stress and Cosserat, [40].

\section{Acknowledgements}

This research was supported by Italian Ministry of University and Research: PRIN 2015, project 2015JW9NJT (B86J16002300001); Sapienza Research Grants 'Grandi Progetti' 2016 (B82F16005920005) and 'Progetti Medi' 2017 (B83C17001440005). 


\section{References}

[1] Pingaro, M., Reccia, E., Trovalusci, P., and Masiani, R., 2019. "Fast statistical homogenization procedure (fshp) for particle random composites using virtual element method". Computational Mechanics, https://doi.org/10.1007/s00466018-1665-7.

[2] Stefanou, G., 2009. "The stochastic finite element method: Past, present and future". Computer Methods in Applied Mechanics and Engineering, 198(9-12), pp. 1031-1051.

[3] Charmpis, D. C., Schuëller, G. I., and Pellissetti, M. F., 2007. "The need for linking micromechanics of materials with stochastic finite elements: A challenge for materials science". Computational Materials Science, 41(1), pp. 27-37.

[4] Stefanou, G., Savvas, D., and Papadrakakis, M., 2015. "Stochastic finite element analysis of composite structures based on material microstructure". Composite Structures, 132, pp. 384-392.

[5] Ostoja-Starzewski, M., 2006. "Material spatial randomness: From statistical to representative volume element". Probabilistic Engineering Mechanics, 21, pp. 112-132.

[6] Sadowski, T., and Marsavina, L., 2011. "Multiscale modelling of two-phase ceramic matrix composites". Computational Materials Science, 50(4), pp. 1336 - 1346.

[7] Ma, J., Sahraee, S., Wriggers, P., and De Lorenzis, L., 2015. "Stochastic multiscale homogenization analysis of heterogeneous materials under finite deformations with full uncertainty in the microstructure". Computational Mechanics, 55(5), pp. 819-835.

[8] Ghosh, S., and Kubair, D. V., 2016. "Exterior statistics based boundary conditions for representative volume elements of elastic composites". Journal of the Mechanics and Physics of Solids, 95, pp. $1-24$.

[9] Savvas, D., and Stefanou, G., 2017. "Assessment of the effect of microstructural uncertainty on the macroscopic properties of random composite materials". Journal of Composite Materials, 51(19), pp. 2707-2725.

[10] Ramakrishnan, N., and Arunachalam, V. S., 1990. "Effective elastic moduli of porous solids". Journal of Materials Science, 25(9), pp. 3930-3937.

[11] Said, B. M., Salah, M., Kanit, T., and Kamel, F., 2016. "On the homogenization of 2d porous material with determination of rve". International Journal of Mechanical and Mechatronics Engineering, 16(1), pp. 81-86.

[12] Herakovich, C. T., and Baxter, S. C., 1999. "Influence of pore geometry on the effective response of porous media". Journal of Materials Science, 34(7), pp. 1595-1609.

[13] Roberts, A. P., and Garboczi, E. J., 2000. "Elastic properties of model porous ceramics". Journal of the American Ceramic Society, 83(12), pp. 3041-3048.

[14] Poutet, J., Manzoni, D., Hage-Chehade, F., Jacquin, C. J., Boutca, M. J., Thovert, J. ., and Adler, P. M., 1996. "The effective mechanical properties of random porous media". Journal of the Mechanics and Physics of Solids, 44(10), pp. 1587-1620.

[15] Biot, M. A., 1941. "General theory of three-dimensional consolidation”. Journal of Applied Physics, 12(2), pp. 155164.

[16] Coussy, O., 1991. Mcanique des milieux poreux. Editions Technip.

[17] Coussy, O., 2010. Mechanics and Physics of Porous Solids. Mechanics and Physics of Porous Solids. John Wiley \& Sons, Ltd.

[18] Sciarra, G., dell'Isola, F., and Coussy, O., 2007. "Second gradient poromechanics". International Journal of Solids and Structures, 44(20), pp. 6607-6629.

[19] Sanchez-Palencia, E., 1980. Non-Homogeneous Media and Vibration Theory, Vol. 127 of Lecture Notes in Physics. Springer-Verlag Berlin Heidelberg.

[20] Ghosh, S., Lee, K., and Raghavan, P., 2001. "A multi-level computational model for multi-scale damage analysis in composite and porous materials". International Journal of Solids and Structures, 38(14), pp. 2335-2385.

[21] Geers, M. G. D., Kouznetsova, V. G., and Brekelmans, W. A. M., 2010. "Multi-scale computational homogenization: Trends and challenges". Journal of Computational and Applied Mathematics, 234(7), pp. 2175-2182.

[22] Ostoja-Starzewski, M., 2007. Microstructural Randomness and Scaling in Mechanics of Materials. CRC Press, Taylor \& Francis Group.

[23] Trovalusci, P., Ostoja-Starzewski, M., De Bellis, M. L., and Murrali, A., 2015. "Scale-dependent homogenization of random composites as micropolar continua". European Journal of Mechanics A/Solids, 49, pp. 396-407.

[24] Kanit, T., Forest, S., Galliet, I., Mounoury, V., and Jeulin, D., 2003. "Determination of the size of the representative volume element for random composites: statistical and numerical approach". International Journal of Solids and Structures, 40(1314), pp. $3647-3679$.

[25] Du, X., and Ostoja-Starzewski, M., 2006. "On the size of representative volume element for darcy law in random media". Proceedings of the Royal Society of London A: Mathematical, Physical and Engineering Sciences, 462(2074), pp. 2949-2963.

[26] Khisaeva, Z. F., and Ostoja-Starzewski, M., 2006. "On the size of rve in finite elasticity of random composites". Journal of Elasticity, 85(2), Aug, p. 153.

[27] Gitman, I. M., Askes, H., and Sluys, L. J., 2007. "Representative volume: Existence and size determination". Engi- 
neering Fracture Mechanics, 74(16), pp. 2518-2534.

[28] Zeman, J., and Sejnoha, M., 2007. "From random microstructures to representative volume elements". Modelling and Simulation in Materials Science and Engineering, 15(4), pp. S325-S335.

[29] Ranganathan, S. I., and Ostoja-Starzewski, M., 2008. "Scaling function, anisotropy and the size of rve in elastic random polycrystals". Journal of the Mechanics and Physics of Solids, 56, pp. 2773-2791.

[30] Ranganathan, S. I., and Ostoja-Starzewski, M., 2009. "Towards scaling laws in random polycrystals". International Journal of Engineering Science, 47(11), pp. 1322 - 1330. Mechanics, Mathematics and Materials a Special Issue in memory of A.J.M. Spencer FRS.

[31] Bouaoune, L., Brunet, Y., Moumen, A. E., Kanit, T., and Mazouz, H., 2016. "Random versus periodic microstructures for elasticity of fibers reinforced composites". Composites Part B: Engineering, 103, pp. 68 - 73.

[32] Djebara, Y., Moumen, A. E., Kanit, T., Madani, S., and Imad, A., 2016. "Modeling of the effect of particles size, particles distribution and particles number on mechanical properties of polymer-clay nano-composites: Numerical homogenization versus experimental results". Composites Part B: Engineering, 86, pp. 135 - 142.

[33] Sadowski, T., and Pankowski, B., 2016. "Numerical modelling of two-phase ceramic composite response under uniaxial loading”. Composite Structures, 143, pp. 388 - 394.

[34] Savvas, D., Stefanou, G., and Papadrakakis, M., 2016. "Determination of rve size for random composites with local volume fraction variation”. Computer Methods in Applied Mechanics and Engineering, 305, pp. 340-358.

[35] Kubair, D. V., Pinz, M., Kollins, K., Przybyla, C., and Ghosh, S., 2018. "Role of exterior statistics-based boundary conditions for property-based statistically equivalent representative volume elements of polydispersed elastic composites". Journal of Composite Materials, 52(21), pp. 2919-2928.

[36] Trovalusci, P., De Bellis, M. L., Ostoja-Starzewski, M., and Murrali, A., 2014. "Particulate random composites homogenized as micropolar materials". Meccanica, 49(11), Nov, pp. 2719-2727.

[37] Reccia, E., De Bellis, M. L., Trovalusci, P., and Masiani, R., 2018. "Sensitivity to material contrast in homogenization of random particle composites as micropolar continua". Composites Part B: Engineering, 136, pp. 39 - 45.

[38] Hill, R., 1963. "Elastic properties of reinforced solids: Some theoretical principles". Journal of the Mechanics and Physics of Solids, 11(5), pp. $357-372$.

[39] Trovalusci, P., De Bellis, M., and Ostoja-Starzewski, M., 2016. "A statistically-based homogenization approach for particle random composites as micropolar continua". In Generalized Continua as Models for Classical and Advanced Materials, H. Altenbach and S. Forest, eds., Vol. 42 of Advanced Structured Materials. Springer International Publishing, pp. 425-441.

[40] Trovalusci, P., De Bellis, M. L., and Masiani, R., 2017. “A multiscale description of particle composites: From lattice microstructures to micropolar continua". Composites Part B: Engineering, 128, pp. 164 - 173.

[41] Salmi, M., Auslender, F., Bornert, M., and Fogli, M., 2012. "Apparent and effective mechanical properties of linear matrix-inclusion random composites: Improved bounds for the effective behavior". International Journal of Solids and Structures, 49(10), pp. 1195 - 1211.

[42] Beiro Da Veiga, L., Brezzi, F., Cangiani, A., Manzini, G., Marini, L. D., and Russo, A., 2013. "Basic principle of virtual element methods". Mathematical Models and Methods in Applied Sciences, 23(01), pp. 199-214.

[43] Beiro Da Veiga, L., Brezzi, F., and Marini, L., 2013. "Virtual elements for linear elasticity problems". SIAM Journal on Numerical Analysis, 51(2), pp. 794-812.

[44] Gain, A. L., Talischi, C., and Paulino, G. H., 2014. "On the virtual element method for three-dimensional linear elasticity problems on arbitrary polyhedral meshes". Computer Methods in Applied Mechanics and Engineering, 282, pp. 132-160.

[45] Wriggers, P., and Hudobivnik, B., 2017. "A low order virtual element formulation for finite elasto-plastic deformations". Computer Methods in Applied Mechanics and Engineering, 327, pp. 459-477.

[46] De Bellis, M. L., Wriggers, P., Hudobivnik, B., and Zavarise, G., 2018. "Virtual element formulation for isotropic damage". Finite Elements in Analysis and Design, 144, pp. 38 - 48.

[47] Artioli, E., Marfia, S., and Sacco, E., 2018. "High-order virtual element method for the homogenization of long fiber nonlinear composites". Computer Methods in Applied Mechanics and Engineering, 341, pp. 571-585.

[48] Ramakrishnan, N., and Arunachalam, V. S., 1993. "Effective elastic moduli of porous ceramic materials". Journal of the American Ceramic Society, 76(11), pp. 2745-2752.

[49] Arnold, M., Boccaccini, A. R., and Ondracek, G., 1996. "Prediction of the poisson's ratio of porous materials". Journal of Materials Science, 31(6), pp. 1643-1646.

[50] Asmani, M., Kermel, C., Leriche, A., and Ourak, M., 2001. "Influence of porosity on youngs modulus and poisson's ratio in alumina ceramics". Journal of the European Ceramic Society, 21(8), pp. 1081-1086.

[51] Artioli, E., Beiro Da Veiga, L., Lovadina, C., and Sacco, E., 2017. "Arbitrary order 2d virtual elements for polygonal meshes: part i, elastic problem”. Computational Mechanics, 60(3), pp. 355-377. 